Canadian Journal of Medicine

WWW.CIKD.CA

\title{
Metastatic Poorly Differentiated Carcinoma of Unknown Primary Site
}

\author{
Zahra Sadeghi $^{1}$, Hossein Pakzad $^{2}$, Hamide Rahmani seraji ${ }^{3 *}$ \\ ${ }^{1}$ Department of Genetic, Tehran-North branch, Islamic Azad university, Tehran, Iran \\ ${ }^{2}$ Department of Biology, Tehran-east branch, Islamic Azad university, Tehran, Iran \\ ${ }^{3}$ Department of Oncology and Hematology, ayatollah Taleghani hospital, Tehran, Iran
}

\section{Keywords:}

Adrenal mass, Metastasis, Undifferentiated carcinoma, Unknown Primary Carcinoma

\section{Received}

06 May 2020

Received in revised form

02 June 2020

Accepted

20 June 2020

\begin{abstract}
Cancer of Unknown Primary (CUP) is a malignant widespread metastatic disease, which primary lesion cannot be identified by exact clinical Inspection. We reported a case of metastases from cancer of unknown primary here. A 58-year-old male presented with abdominal pain, backache, weakness and weight loss. Abdominopelvic sonography of the patient revealed two heterogeneous mass lesions in the right and left of the adrenal region. The primary site of the tumor was not identified According to our evaluation. We performed brain magnetic resonance imaging (CT) for the patient that showed a very large mainly peripheral enhancing mass lesion measuring $48 \times 37 \mathrm{~mm}$ in the cerebellum as Glioblastoma multiforme (GBM). We did chemotherapy with platinum-based regimens including paclitaxel and carboplatin. In this case, the clinical situation of the patient swiftly deteriorated and unfortunately before doing local treatment for the brain mass we lost our case.
\end{abstract}

*Correspondence:

rahamaniseraji63.hr@gmail.com

(C)CIKD Publishing

\section{Introduction}

Cancer of unknown primary site (CUP) is a heterogeneous group of metastatic tumors, accounting for 4 to 5 percent of all invasive cancers [1]. In this category, tumors from many primary sites with varying biology are illustrated. Symptoms of the patients with CUP are related to the respective organ involvement and the extent of metastasis. The initial evaluation, including physical examination, laboratory studies, and imaging, often cannot find the primary site. Diagnosis of CUP requires pathology evaluation and Immunohistochemistry [2]. The affected organs in CUP include: Lymph nodes (40-45\%), liver (30-40\%), Skeleton (25-35\%), Lung (30-40\%), Pleura (5-15\%), 
Peritoneum (5- 10\%), Central nervous system (5-10\%), Adrenal glands (6\%) and Skin (4\%) [3]. Brain metastasis in CUP is not rare as $15 \%$ of the patients with brain metastases, lacking a primary tumor [4]. The clinical picture of brain metastasis in CUP is similar to primary brain tumors but seems to differ in the areas of predilection for metastasis in the brain parenchyma [4]. But sometimes, differentiation of primary brain tumors is from the secondary ones. The primary central nervous system malignancies are relatively uncommon kinds of malignancies which account for less than $2 \%$ of overall malignancies [5] and Glioblastoma multiform (GBM) is the most common malignant primary brain tumor in adults. Extracranial metastasis is rare and frequent in the lungs, the posterior cervical soft tissue, and the lumbar intradural space [6]. In this manuscript, we present a case of CUP with bilateral adrenal masses and pulmonary nodules and a brain mass.

\section{Case Presentation}

A 58-year-old man was evaluated in the hospital due to abdominal pain, backache, weakness and weight loss. The patient had a history of myocardial infarction and severe heart failure (ejection fraction: 20\%), Tourette syndrome and light headache over the past few months. The patient had a history of two pack-years smoking from about 20 years ago and had no history of any medication

\section{Investigations}

Abdominopelvic sonography of the patient revealed two heterogeneous mass lesions in right and left the adrenal area with a size of $80 \times 44 \mathrm{~mm}$ and $61 \times 40 \mathrm{~mm}$ respectively. Initial laboratory tests showed a normal $1 \mathrm{mg}$ overnight dexamethasone suppression test, normal 4 24-hour urinary fractionated metanephrines and catecholamines and normal plasma aldosterone concentration and plasma renin activity. Hemoglobin, platelets, routine serum chemistry determinations, liver function test, serum-free and total PSA, human chorionic gonadotropin (hCG) and alphafetoprotein (AFP) level were normal. A computed tomography scan of the abdomen and pelvic with intravenous contrast demonstrated bilateral mild enhancing mass lesions at both adrenal areas, suggestive of primary mass lesions versus metastatic lesions (Figure 1), also multiple pulmonary nodules was seen in chest computerized tomography (CT) scan of the patient (Figure 2).

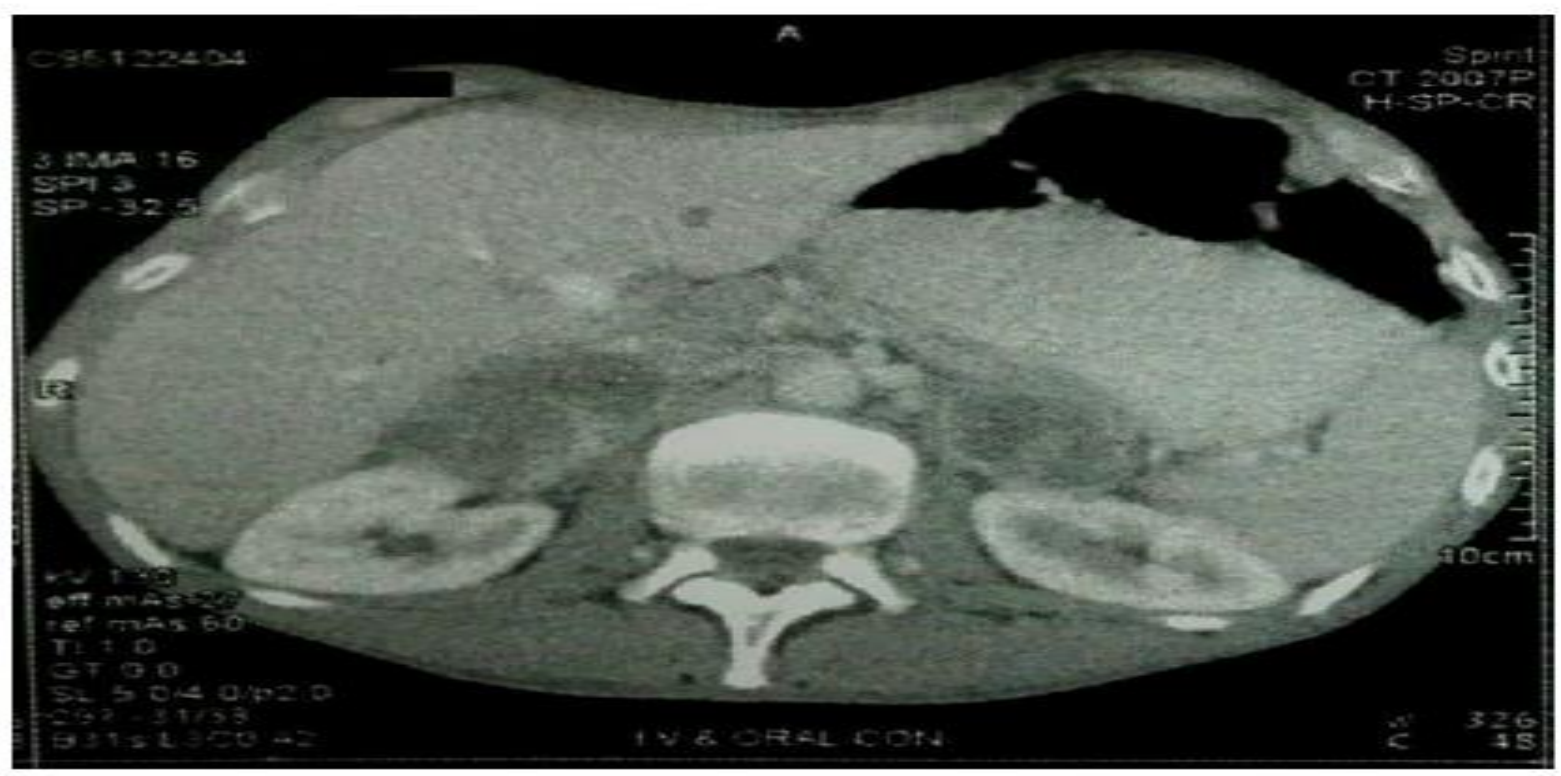

Figure 1. CT scan of the adrenal areas, shows bilateral mass lesion 


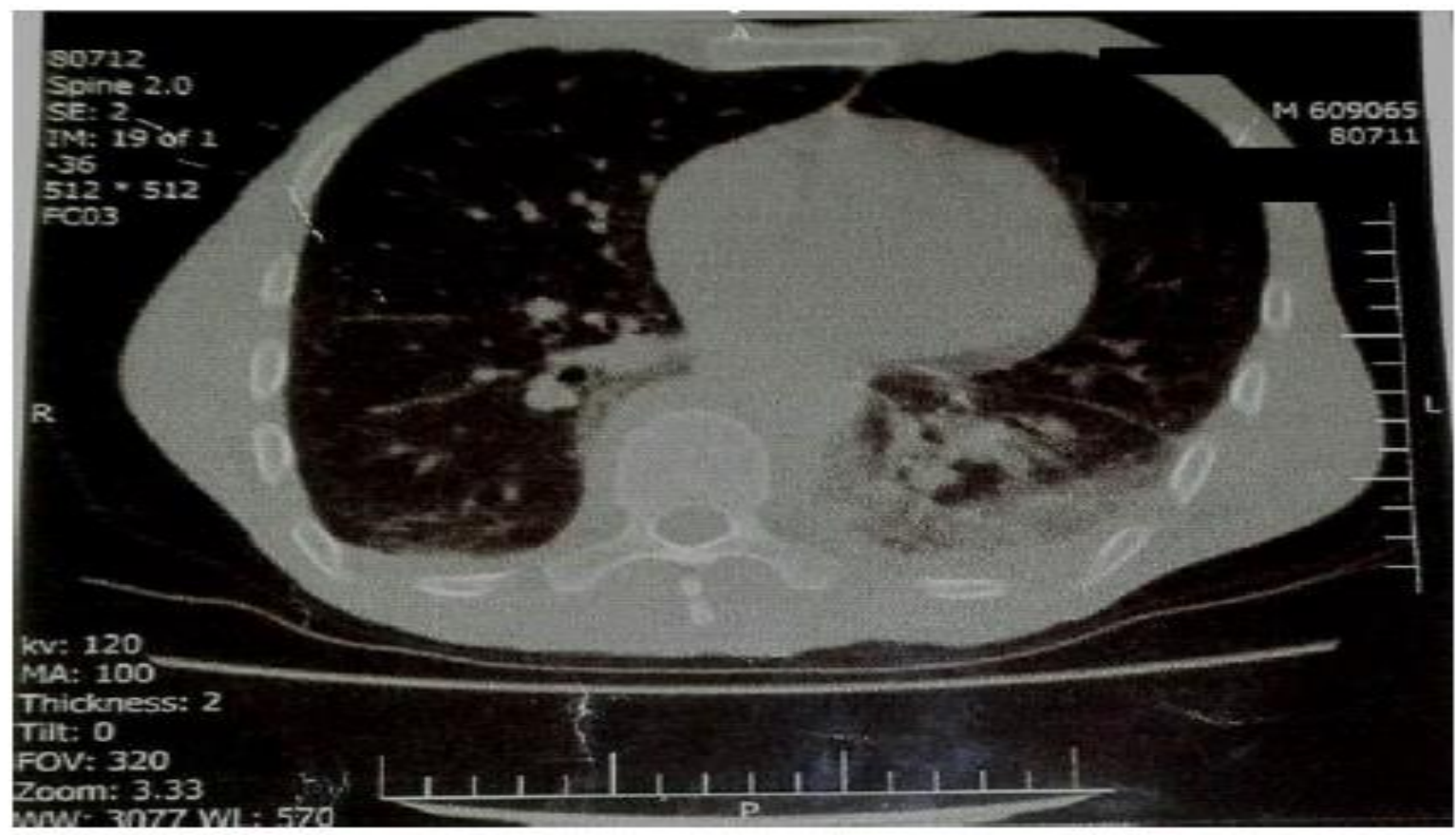

Figure 2. CT scan of the lungs, shows multiple nodule

\section{Treatment}

Fine needle biopsy in the guide of CT scan was performed for the patient and also immunohistochemistry was done on the specimen. One week after that, immunohistochemistry (IHC) study results showed metastatic undifferentiated carcinoma based on (TTF1: negative, CK7: positive, CK20: negative, Heppar1: negative, CDX: negative, PSA: negative, Chromogranine: negative, Synaptophysin: negative, Calretinin: negative, LCA: negative, CD8: negative, Ki67: positive in $60 \%$ of tumor cell). The primary site of the tumor could not be specified according to our evaluation so we decided to initiation the chemotherapy with platinum-based regimens and we prescribed carboplatin and paclitaxel for the patient. Two days later the patient abruptly lost his consciousness and we performed brain magnetic resonance imaging (MRI) for the case that it showed very large mainly peripheral enhancing mass measuring $48 \times 37 \mathrm{~mm}$ in the cerebellum which crosses the midline and most compatible with a malignant lesion as GBM. It seems that the mentioned mass has hemorrhagic components as the hyper signal intensity in T1. Lateral ventricles were dilated (Figure 3). Intravenous dexamethasone quickly was administered for the patient and Surgery and radiotherapy consults were done for the case, but unluckily we lost our patient before we can therapeutic intervention. 


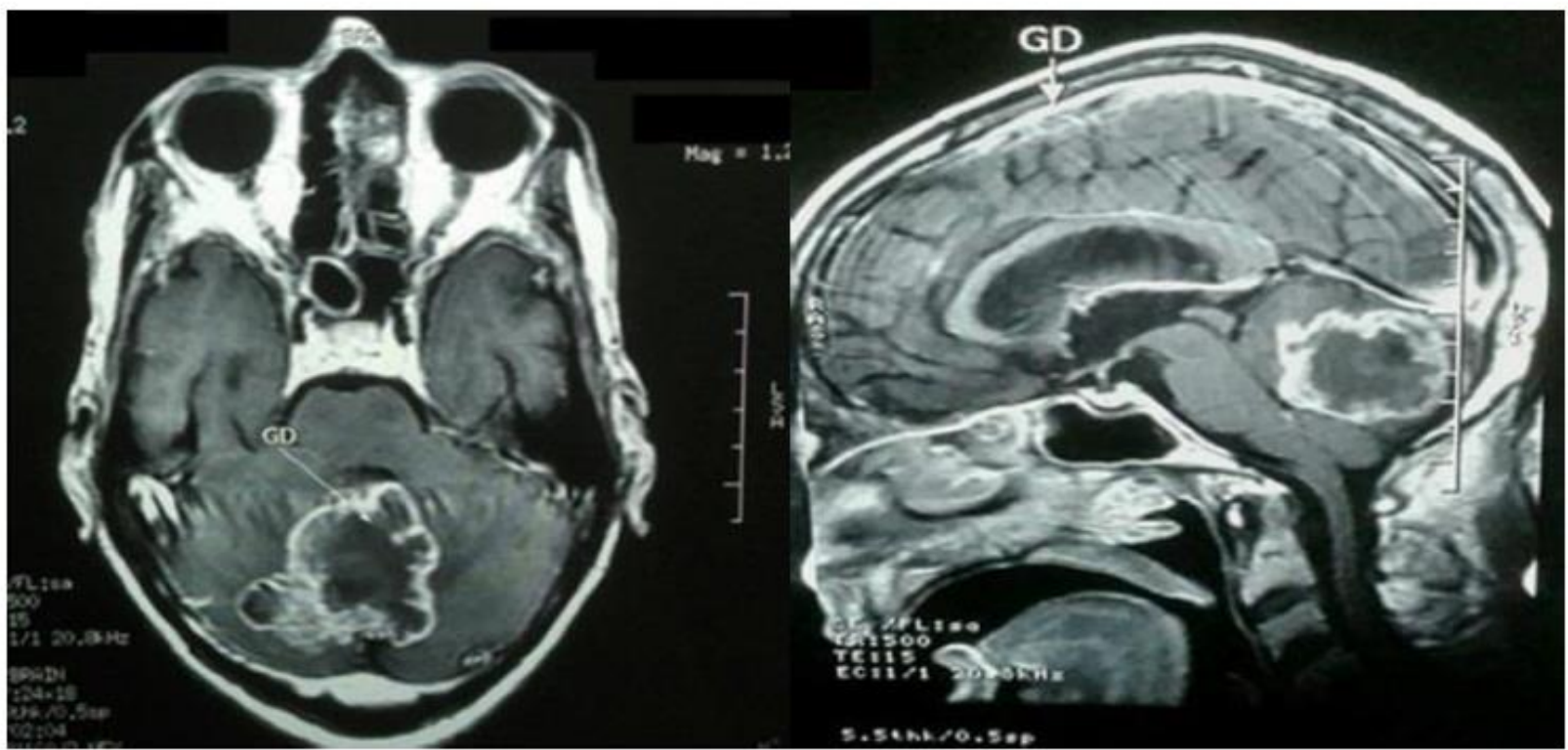

Figure 3. MRI with contrast, shows a large mass as a GBM

\section{Results}

In this case, we just could initial chemotherapy and local treatment. The brain biopsy, rebiopsy of adrenal and other investigations were not done, because his family did not allow due to the history of severing heart failure. The patient rejected colonoscopy and endoscopy too. Unfortunately, the clinical condition of the case deteriorated rapidly and 5 we lost the patient and his family did not agree to perform a brain biopsy after the patient's death.

\section{Discussion}

CUP is a metastatic disease without an established primary site which accounts for about 4-5 percent of all invasive cancers [7]. About 25 percent of CUPs are poorly differentiated and cannot be characterized by histological examination. Nearby 80 percent of these poorly differentiated tumors have features of carcinoma and are named poorly differentiated carcinoma. A wide variety of primary sites exist for these cancers and most frequently identified sites are lung, pancreas, hepatobiliary tree, and kidney [8]. Finding the initial site of the tumor as accurate as possible is essential for the selection of the best treatment for the patient however in 20 to 30 percent of cases, the primary site cannot be found [8]. The initial diagnostic evaluation consists of history, physical examination, and routine laboratory testing, imaging (CT or MRI of the chest, abdomen, and pelvis), Serum levels of human chorionic gonadotropin (hCG) and alpha-fetoprotein (AFP) should be obtained in all patients with CUP. In our patient, CT scan of the chest and abdomen revealed multiple pulmonary nodules and bilateral mass lesions in adrenals which histological examination of the adrenal mass demonstrated undifferentiated carcinoma. Functional tests of adrenal, hCG, AFP and other laboratory tests were normal. Examination of poorly differentiated malignancies using routine light microscopy alone is inadequate and IHC is precious in identifying the tumor type and primary site. For example, Positive staining for cytokeratins provides strong evidence for a carcinoma, positive staining for chromogranin A or synaptophysin is suggestive of neuroendocrine carcinoma [9-11], and positive staining for PSA is specific for prostate cancer [12]. Of course, panels of IHC stains are more effective than a single [13-15]. In our patient TTF1 and CK20 were negative, and CK7 was positive which this panel was inconsistent with the diagnosis of lung adenocarcinoma. PSA, CDX, Heppar1, Chromogranine, and Synaptophysin were also negative therefore prostate carcinoma, colorectal carcinoma, pancreas carcinoma, hepatocellular carcinoma, and neuroendocrine carcinoma were ruled out. As well as immunostaining, LCA in our 
case was negative that could preclude non-hodgkin lymphomas and hodgkin lymphomas [16]. So, the primary site of the tumor was not identified based on our evaluation. We initiated 6 chemotherapy with platinum-based regimens including carboplatin and paclitaxel. Two days later, our patient suddenly lost consciousness. Brain MRI was done for the case quickly which demonstrated a large mass lesion measuring $48 \times 37 \mathrm{~mm}$ in the cerebellum with hemorrhagic components. Surgery consult was done for the case but the surgeon refused surgery because the patient was a known case of severe heart failure with ejection fraction $20 \%$ and the risk of mortality for the case was very high, so the patient was a candidate for radiotherapy but unfortunately died before radiotherapy was done. CUP with brain metastasis is an unfavorable subset of the CUP which is a very aggressive disease but brain metastasis does not seem to hurt the survival of the patients with known versus unknown primary sites and is seen predominantly in male patients in the sixth decade of age [4]. Tissue diagnosis in this setting is essential especially in those patients with a solitary lesion similar to our case. The delay in treatment, until recognition of a primary site of the tumor, is not reasonable [4]. Treatment of the brain metastasis in CUP is similar to the management of brain metastases with known primary tumors and the published literature about the management of brain metastasis in CUP is limited to small historical series. Complete tumor resection is a cornerstone in the management of patients with resectable tumors and less than four lesions. For patients with more than four or unresectable lesions, whole-brain irradiation is recommended. Systemic chemotherapy had limited benefits in these patients same to case with brain metastases from a known primary site [4].

\section{Conclusion}

Brain mass as a primary site of the tumor can be raised, although the probability is low because extracranial metastasis of primary central nervous system malignancies, that GBM is the most common type of these tumors, is rare and it is seen most frequently in the lungs, the posterior cervical soft tissue, and the lumbar intradural space. Another possibility, that may be more relevant to the patient as regards the concurrent involvement of the lung, adrenal, and the brain, is that the lung is the primary site of the tumor. But none of these assumptions can be proven especially we do not have tissue diagnosis from brain mass however identification of primary sites in these settings had not to impact survival.

\section{Acknowledgement}

Special thanks to personnel of ayatollah Taleghani hospital of Tehran

\section{Funding}

The authors have not declared a specific grant for this research from any funding agency in the public, commercial or not-for-profit sectors.

\section{Competing of interests}

None declared 


\section{List of abbreviations}

$\begin{array}{ll}\text { AFP } & \text { Alpha-Fetoprotein } \\ \text { CT } & \text { Computerized Tomography } \\ \text { CUP } & \text { Cancer of Unknown Primary } \\ \text { GBM } & \text { Glioblastoma Multiform } \\ \text { hCG } & \text { Human Chorionic Gonadotropin } \\ \text { IHC } & \text { Immunohistochemistry } \\ \text { MRI } & \text { Magnetic Resonance Imaging }\end{array}$

\section{References}

[1] Greco FA, Hainsworth JD. Cancer of unknown primary site. Oncology: Springer. 2006;1119- 32.

[2] Oien KA, editor Pathologic evaluation of unknown primary cancer. Seminars in Oncology. 2009 Mar; 36(1):8-37.

[3] Zaun G, Schuler M, Herrmann K, Tannapfel A. CUP syndrome-metastatic malignancy with unknown primary tumor. 2018 Mar;115(10):157.

[4] Rassy E, Zanaty M, Azoury F, Pavlidis NJFO. Advances in the management of brain metastases from cancer of unknown primary. 2019;15(23):2759-68.

[5] Singal R, Agarwal A, Khurana A, Bhasin A, Kirtani P, Vanjara W. Glioblastoma multiforme with gliosarcoma with metastasis to lung. 2018 Jun;9(1):40-3.

[6] Türkeş G, Parmaksız ET, Kıral N, Doğan C, Sağmen SB, Fidan A, Cömert S. A rare cause of lung metastasis-glioblastoma multiforme. Southern Clinics of Istanbul Eurasia. 2018 Sep 1;29(3).

[7] Greco FA, Hainsworth JD. Introduction: Unknown primary cancer. Seminars in Oncology; 2009; 1(36):6-7.

[8] Pentheroudakis G, Golfinopoulos V, Pavlidis N. Switching benchmarks in cancer of unknown primary: from autopsy to microarray. European Journal of Cancer. 2007 Sep 1;43(14):2026-2036.

[9] Denk H, Krepler R, Artlieb U, Gabbiani G, Rungger-Brändle E, Leoncini P, Franke WW. Proteins of intermediate filaments. An immunohistochemical and biochemical approach to the classification of soft tissue tumors. The American journal of pathology. $1983 \mathrm{Feb} ; 110(2): 193-298$.

[10] Osborn M, Weber K. Tumor diagnosis by intermediate filament typing: a novel tool for surgical pathology. Laboratory investigation; a Journal of Technical Methods and Pathology. 1983 Apr;48(4):372-94.

[11] Ramaekers FC, Vroom TM, Moesker O, Kant A, Scholte G, Vooijs GP. The use of antibodies to intermediate filament proteins in the differential diagnosis of lymphoma versus metastatic carcinoma. The histochemical journal. $1985 \mathrm{Jan}$ 1;17(1):57-70.

[12] Allhoff EP, Proppe KH, Chapman CM, Lin CW, Prout GR. Evaluation of prostate specific acid phosphatase and prostate specific antigen in identification of prostatic cancer. The Journal of Urology. 1983 Feb;129(2):315-8.

[13] Park SY, Kim BH, Kim JH, Lee S, Kang GH. Panels of immunohistochemical markers help determine primary sites of metastatic adenocarcinoma. Archives of Pathology \& Laboratory Medicine. 2007 Oct;131(10):1561-7.

[14] Anderson GG, Weiss LM. Determining tissue of origin for metastatic cancers: meta-analysis and literature review of immunohistochemistry performance. Applied Immunohistochemistry \& Molecular Morphology. 2010 Jan 1;18(1):3-8.

[15] Oien KA, Dennis JL. Diagnostic work-up of carcinoma of unknown primary: from immunohistochemistry to molecular profiling. Annals of Oncology. 2012 Sep 1;23(suppl_10):x271-7.

[16] Michels S, Swanson PE, Frizzera G, Wick MR. Immunostaining for leukocyte common antigen using an amplified avidinbiotin-peroxidase complex method and paraffin sections. A study of 735 hematopoietic and nonhematopoietic human neoplasms. Archives of pathology \& Laboratory Medicine. 1987 Nov;111(11):1035-9. 